\title{
Should mesenteric tumor deposits be included in staging of well-differentiated small intestine neuroendocrine tumors?
}

\author{
Raul S Gonzalez ${ }^{1}$, Eric H Liu ${ }^{2}$, JoAnn R Alvarez ${ }^{3}$, Gregory D Ayers ${ }^{3}$, M Kay Washington ${ }^{1}$ \\ and Chanjuan Shi ${ }^{1}$ \\ ${ }^{1}$ Department of Pathology, Microbiology and Immunology, Vanderbilt University Medical Center, Nashville, \\ TN, USA; ${ }^{2}$ Department of Surgery, Vanderbilt University Medical Center, Nashville, TN, USA and \\ ${ }^{3}$ Department of Biostatistics, Vanderbilt University Medical Center, Nashville, TN, USA
}

\begin{abstract}
Well-differentiated small intestine neuroendocrine tumors can give rise to mesenteric tumor deposits, which are not included in the current American Joint Committee on Cancer staging system for small intestine neuroendocrine tumors, and their impact on patient prognosis is unknown. Seventy-two small intestine neuroendocrine tumors resections were identified in our files with slides, reports, and follow-up data available. Cases were assessed for T-category and for the presence of mesenteric tumor deposits, lymph node metastases, lymphovascular invasion, and liver metastases. Mesenteric tumor deposits were defined as discrete mesenteric tumor nodules $\geq 1 \mathrm{~mm}$ with an irregular growth profile. Similar lesions clearly resulting from extranodal extension or direct contiguous spread by the primary lesion were excluded. Forty-three of the 72 cases had mesenteric tumor deposits (60\%). The deposits were significantly associated with lymphovascular invasion $(P=0.001)$, pT3 or pT4 disease $(P=0.001)$, nodal metastases $(P=0.040)$, and liver metastases $(P<0.001)$ at the time of surgery. In addition, four of six cases with tumor deposits and no nodal disease had liver disease. Tumor deposits were associated with an increased incidence of disease progression and death due to the disease $(\boldsymbol{P}=\mathbf{0 . 0 0 1})$. Finally, the presence of tumor deposits at the time of surgery was associated with an increase in hazard of progression or death due to disease (hazard ratio: 4.0; 95\% confidence interval: 1.3, 12.5; $P=0.016)$. Mesenteric tumor deposits are present in the majority of cases of small intestine neuroendocrine tumors and are indicators of poor prognosis for this disease. Therefore, they may have a place in staging of small intestine neuroendocrine tumors, perhaps as analogous to lymph node disease. Modern Pathology (2014) 27, 1288-1295; doi:10.1038/modpathol.2013.232; published online 24 January 2014
\end{abstract}

Keywords: mesentery; small intestine; staging; well-differentiated neuroendocrine tumors

Well-differentiated small intestine (midgut) neuroendocrine tumors are the second most common primary malignancy in the small intestine, ${ }^{1}$ and the small intestine is the most common site for neuroendocrine tumors in the gastrointestinal tract. ${ }^{2}$ Unless detected early, small intestine neuroendocrine tumors can have a protracted clinical course; jejunal cases, for example, are capable of metastasizing before reaching a primary size of $1 \mathrm{~cm}$, and nearly all lesions $>1 \mathrm{~cm}$ metastasize. ${ }^{3}$ Known

Correspondence: Dr C Shi, MD, PhD, Department of Pathology, Microbiology and Immunology, Vanderbilt University School of Medicine, 1161, 21st Avenue South, C-3321 MCN, Nashville, TN 37232-2561, USA.

E-mail: chanjuan.shi@vanderbilt.edu

Received 26 August 2013; revised 22 October 2013; accepted 23 October 2013; published online 24 January 2014 prognostic factors in small intestine neuroendocrine tumors include location, size, and proliferative rate as assessed by mitotic figure count or Ki-67 immunohistochemical staining. ${ }^{3}$ The tumors often metastasize to the liver during their clinical course, ${ }^{4}$ and the 5-year survival rate is roughly $63 \%$, which has remained steady for the past few decades. ${ }^{5}$

Although widespread liver metastasis is the most common cause of cancer-related death for patients with small intestine neuroendocrine tumors, some patients die from small bowel ischemia and infarct due to extensive mesenteric disease. ${ }^{6}$ Mesenteric disease in patients with small intestine neuroendocrine tumors develops from tumor-involved mesenteric lymph nodes and/or extranodal mesenteric tumor deposits. Careful macroscopic and microscopic examination of the mesentery in small intestinal resections for small intestine neuroendocrine tumor 
can reveal the presence of mesenteric tumor deposits. Although such tumor deposits are a well-recognized prognostic factor in colorectal carcinoma ${ }^{7-10}$ and are included in the current American Joint Committee on Cancer staging summary for that entity, ${ }^{11}$ tumor deposits in small intestine neuroendocrine tumors have undergone much less scrutiny and may be erroneously interpreted as lymph nodes harboring metastatic disease by surgeons, radiologists, and pathologists. Whether they function as harbingers of lymph node or liver metastases, or as independent prognostic factors affecting survival, is currently not known.

In the present study, we evaluated the presence and significance of mesenteric tumor deposits in patients with small intestine neuroendocrine tumors by reviewing patients' clinical history, imaging studies, and resection specimens.

\section{Materials and methods}

With appropriate Institutional Review Board approval, we searched the archives of the Department of Pathology, Microbiology, and Immunology for resected cases of small intestine neuroendocrine tumors for the time period 17 October 1990 to 30 June 2012. Inclusion criteria included available hematoxylin and eosin-stained slides, pathology reports with staging information, and follow-up data including imaging reports, clinical and pathological metastases, and survival.

Seventy-two cases met these inclusion criteria. All available slides were reviewed for the presence of mesenteric tumor deposits by two pathologists (RSG and CS), with discrepancies resolved through in-person consensus. All cases contained at least some mesenteric tissue. For the purpose of this study, mesenteric tumor deposits were defined as discrete mesenteric tumor nodules $\geq 1 \mathrm{~mm}$ with an irregular growth profile. Tumor deposits often appeared situated next to a large blood vessel, demonstrated an irregular growth pattern, and wrapped around entrapped nerves (Figure 1a). Lymphocytes were typically not present at the periphery of the deposits. Care was taken to distinguish tumor deposits from mesenteric lymph nodes entirely replaced by metastatic disease. Unlike deposits, entirely replaced lymph nodes showed a rounded contour, often with residual lymphoid tissue at the periphery (Figure 1b). There was no consistent association with adjacent blood vessels in replaced lymph nodes, and nerves were usually not present within such lesions. By searching for these morphologic criteria, the differential diagnosis could usually be resolved. In some instances, mesenteric lesions appeared to consist of entirely replaced lymph nodes exhibiting extranodal extension that mimicked the growth pattern of tumor deposits; these were not classified as true deposits in our study, nor were tumor deposit-like lesions directly contiguous with the primary tumor or tumor deposit-like lesions consisting solely of intravascular tumor. On rare occasion, deposits extended locally into lymph nodes.

Pathologic T-category was re-established for all cases based on the American Joint Committee on Cancer seventh edition staging system, excluding mesenteric tumor deposits as a component of tumor size or depth of invasion. Each case was also reviewed for the presence or absence of lymphovascular invasion, lymph node metastases, and liver metastases. When liver disease was present, its extent was noted (ie, single metastasis vs several or innumerable metastases). Ki67 proliferation rate was available for 61 of the 72 cases; this information was used to grade the cases.

Continuous variables were summarized using median and quartiles, and compared among patient groups using the Wilcoxon rank-sum test. Categorical variables were summarized in frequency tables and compared among patient groups using the Pearson $\chi^{2}$-test. Progression-free survival was defined as the time from surgical resection to disease progression, death due to disease, or last contact, whichever came first. Patients alive and progression-free at last contact were censored. Distributions of progression-free survival were estimated using the cumulative incidence function, treating deaths due to other causes as a competing risk and compared among groups using the $\chi^{2}$-statistic of Gray. ${ }^{12}$ Confidence limits for the cumulative incidence were calculated using a log transformation with variance estimators derived by Aalen. ${ }^{13}$ Multivariable models to assess the conditional impact of selected patient characteristics on progression-free survival, with deaths due to other causes also censored, were fitted using the Cox (proportional hazards) regression model. Analyses were conducted using $\mathrm{R}$ version 3.0.1 (R Foundation for Statistical Computing, Vienna, Austria). ${ }^{14}$

\section{Results}

\section{Demographics and Pathology}

Patient demographics are summarized in Table 1. The 72 patients included 39 men and 33 women, with an age range of 19-83 years at resection of primary tumor (median age 59 years). Forty-eight patients $(67 \%)$ had one primary tumor, $8(11 \%)$ had two, and $16(22 \%)$ had three or more. The median size of the largest primary lesion in each patient was $1.7 \mathrm{~cm}$ (range: $0.5-8.0 \mathrm{~cm}$ ). The T-category distribution for the 72 patients was as follows: 3 pT1 (tumor invades lamina propria or submucosa and size $1 \mathrm{~cm}$ or less), 13 pT2 (tumor invades muscularis propria or size $>1 \mathrm{~cm}$ ), 37 pT3 (tumor invades through muscularis propria into subserosal tissue without penetration of overlying serosa), and 19 pT4 (tumor penetrates visceral peritoneum (serosa) or invades 

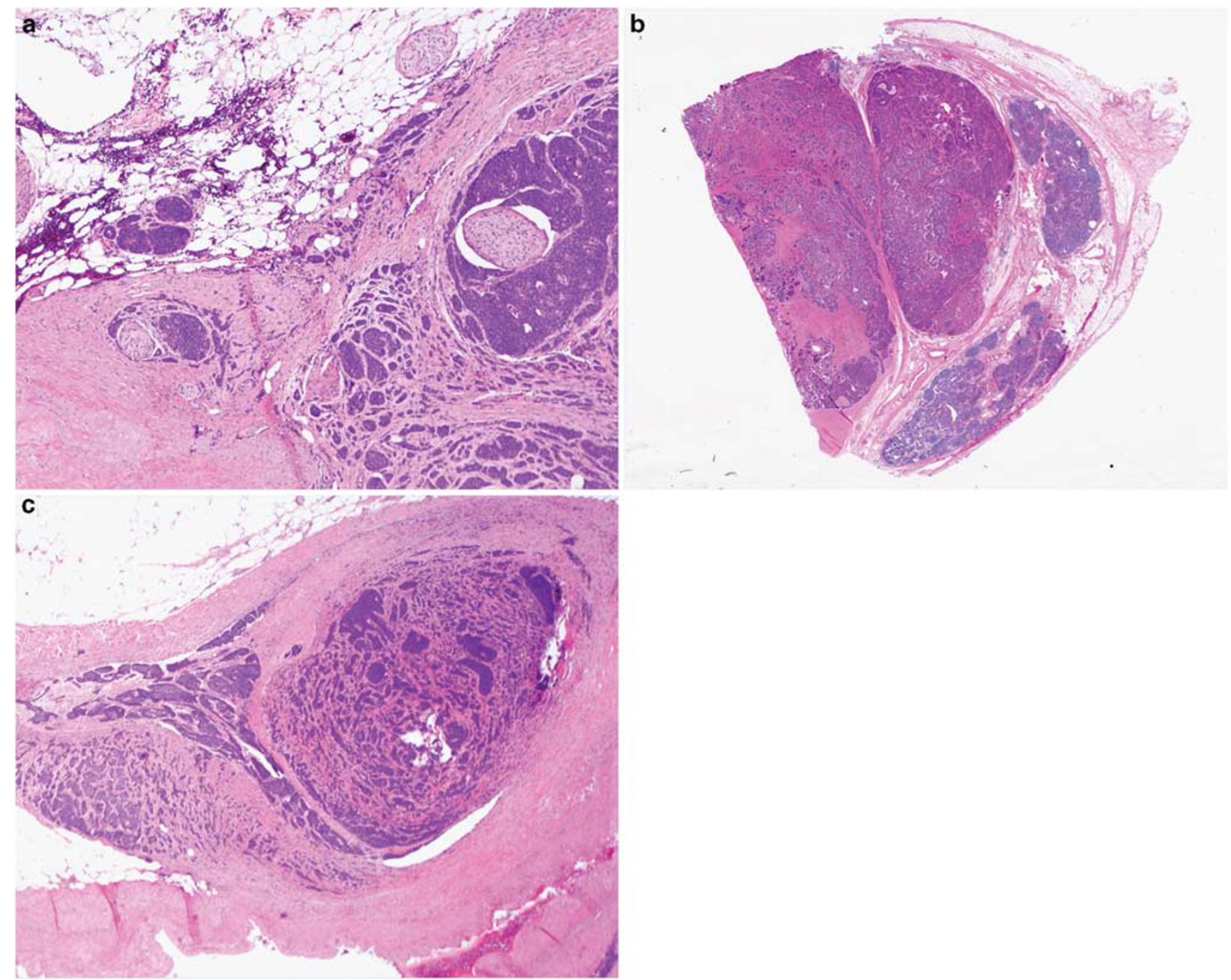

Figure 1 (a) Small intestine neuroendocrine tumor deposit, with numerous entrapped but non-infiltrated nerves. (b) A lymph node involved by metastatic disease, showing peripheral residual lymphoid tissue. (c) A mesenteric tumor deposit next to a vessel harboring metastatic small intestine neuroendocrine tumor in its lumen, strongly suggesting a vascular origin for tumor deposits.

other organs). Additional findings included 58 patients with lymphovascular invasion (81\%), 56 with lymph node metastases $(78 \%), 27$ with liver metastases $(38 \%)$, and 6 with omental tumor implants (8\%).

Two patients had tissue-proven metastases to the ovary, one had multiple lung metastases discovered at autopsy, and one had a sclerotic vertebral lesion radiologically consistent with metastasis; aside from one of the patients with ovarian disease, these patients did not have known distant metastases at time of primary surgery.

\section{Clinicopathologic Features of Mesenteric Tumor Deposits}

Of the 72 cases reviewed, $43(60 \%)$ demonstrated the presence of mesenteric tumor deposits, which ranged in size from $1 \mathrm{~mm}$ to $7.2 \mathrm{~cm}$. In patients with large tumor deposits, additional small-sized deposits $(<1.0 \mathrm{~cm})$ were invariably present. As described above, similar lesions clearly resulting from extranodal extension or direct contiguous spread by the primary lesion were excluded.

The median age was 58 years for the group with no deposits and 60 years for the group with deposits $(P=0.324$; Table 2). The gender distribution was very similar between the two groups $(P=0.533)$. The group with tumor deposits tended to have larger primary tumors than that without deposits; the median tumor size was $1.8 \mathrm{~cm}$ for the former group and $1.5 \mathrm{~cm}$ for the latter group $(P=0.117)$. The incidence of tumor deposits did not appear to be related to the existence of multiple primary tumors $(P=0.734)$.

There were strong associations between the presence of tumor deposits and many other adverse prognostic factors (Table 2). Nearly all patients with deposits had microscopically identifiable 
Table 1 Clinicopathologic data of 72 patients with small intestine neuroendocrine tumor

\begin{tabular}{lr}
\hline $\begin{array}{l}\text { Age at diagnosis (years) } \\
\text { Median }\end{array}$ & 59 \\
Range & $19-83$ \\
& \\
Gender & \\
Male & 39 \\
Female & 33 \\
& \\
Number of tumors & $48(67 \%)$ \\
1 & $8(11 \%)$ \\
2 & $16(22 \%)$ \\
3 or more & \\
& \\
T-category & $3(4 \%)$ \\
pT1 & $13(18 \%)$ \\
pT2 & $37(51 \%)$ \\
pT3 & $19(26 \%)$ \\
pT4 & \\
Lymphovascular invasion present & $58(81 \%)$ \\
Lymph node metastases present & $56(78 \%)$ \\
Liver metastases present & $30(42 \%)$ \\
Progression of liver disease post-op & $24(33 \%)$ \\
& \\
Patient status & \\
Alive, no evidence of disease & $29(40 \%)$ \\
Alive with disease & $23(32 \%)$ \\
Died of disease & $9(12 \%)$ \\
Died of other cause & $11(15 \%)$ \\
\hline
\end{tabular}

lymphovascular invasion (40/43; 93\%), whereas $18 / 29(62 \%)$ patients without deposits had confirmed lymphovascular invasion $(P=0.001)$. Thirty-nine of 43 patients (91\%) with deposits had a T-category of pT3 or pT4 on resection of the primary lesion; of patients without deposits, 17/29 (59\%) had such advanced T-category $(P=0.001)$. Of the 43 patients with deposits, 37 (86\%) had lymph node metastases, whereas 19/29 (66\%) of patients without deposits had nodal metastases $(P=0.04)$. The relationship with liver metastases was even stronger, as $23 / 43$ $(53 \%)$ patients with deposits had liver disease at the time of surgery, compared with only 4/29 (14\%) without tumor deposits $(P<0.001)$.

Of the 61 cases with Ki67 data, 46 (75\%) were grade 1 and the other $15(25 \%)$ were grade 2 , according to the World Health Organization criteria, with Ki67 index $<3 \%$ corresponding to grade 1 and $3-20 \%$ corresponding to grade $2 .{ }^{15}$ Twenty-six of the grade 1 tumors had deposits, whereas 20 lacked them; 11 of the grade 2 tumors showed deposits and 4 did not. There was not a statistically significant correlation between tumor grade and the presence of deposits $(P=0.247)$.

Although lymph node metastases and mesenteric tumor deposits were strongly correlated, some patients demonstrated only one of the two features. Overall, 19 patients had nodal metastases but not deposits, with $4(21 \%)$ of these patients having liver disease. Conversely, 6 patients had deposits but not nodal metastases; 4 (67\%) such patients also had
Table 2 Comparison of small intestine neuroendocrine tumor patients with and without mesenteric tumor deposits

\begin{tabular}{|c|c|c|c|c|}
\hline & $\begin{array}{l}\text { No MTD } \\
(\mathrm{n}=29)\end{array}$ & $\begin{array}{c}M T D \\
(\mathrm{n}=43)\end{array}$ & $\begin{array}{l}\text { Combined } \\
(\mathrm{n}=72)\end{array}$ & $\mathrm{P}$-value \\
\hline Median age (years) & 58 & 60 & 59 & 0.324 \\
\hline Gender & & & & 0.533 \\
\hline Male & $17(59 \%)$ & $22(51 \%)$ & $39(54 \%)$ & \\
\hline Female & $12(41 \%)$ & $21(49 \%)$ & $33(46 \%)$ & \\
\hline Median tumor size & $1.5 \mathrm{~cm}$ & $1.8 \mathrm{~cm}$ & $1.7 \mathrm{~cm}$ & 0.117 \\
\hline T-category & & & & 0.001 \\
\hline $\mathrm{pT} 1 / \mathrm{pT} 2$ & $12(41 \%)$ & $4(9 \%)$ & $16(22 \%)$ & \\
\hline $\mathrm{pT} 3 / \mathrm{pT} 4$ & $17(59 \%)$ & $39(91 \%)$ & $56(78 \%)$ & \\
\hline $\begin{array}{l}\text { Lymphovascular } \\
\text { invasion }\end{array}$ & & & & 0.001 \\
\hline Absent & $11(38 \%)$ & $3(7 \%)$ & $14(19 \%)$ & \\
\hline Present & $18(62 \%)$ & $40(93 \%)$ & $58(81 \%)$ & \\
\hline $\begin{array}{l}\text { Lymph node } \\
\text { metastasis }\end{array}$ & & & & 0.04 \\
\hline Absent & $10(34 \%)$ & $6(14 \%)$ & $16(22 \%)$ & \\
\hline Present & $19(66 \%)$ & $37(86 \%)$ & $56(78 \%)$ & \\
\hline $\begin{array}{l}\text { Number of } \\
\text { primary } \\
\text { tumors }\end{array}$ & & & & 0.734 \\
\hline One & $20(69 \%)$ & $28(65 \%)$ & $48(67 \%)$ & \\
\hline Multiple & $9(31 \%)$ & $15(35 \%)$ & $24(33 \%)$ & \\
\hline $\begin{array}{l}\text { Liver metastasis at } \\
\text { time of surgery }\end{array}$ & & & & $<0.001$ \\
\hline No & $25(86 \%)$ & $20(47 \%)$ & $45(62 \%)$ & \\
\hline Yes & $4(14 \%)$ & $23(53 \%)$ & $27(38 \%)$ & \\
\hline \multicolumn{5}{|l|}{ Grade $^{\mathrm{a}}$} \\
\hline Grade 1 & $\begin{array}{l}20 / 24 \\
(83 \%)\end{array}$ & $\begin{array}{l}26 / 37 \\
(70 \%)\end{array}$ & $\begin{array}{l}46 / 61 \\
(75 \%)\end{array}$ & 0.247 \\
\hline Grade 2 & $\begin{array}{c}4 / 24 \\
(17 \%)\end{array}$ & $\begin{array}{l}11 / 37 \\
(30 \%)\end{array}$ & $\begin{array}{l}15 / 61 \\
(25 \%)\end{array}$ & \\
\hline
\end{tabular}

Abbreviation: MTD, mesentery tumor deposit.

${ }^{a}$ As determined by World Health Organization criteria.

Table 3 Estimated cumulative incidence of progression and death from disease at 2 and 5 years, with $95 \%$ confidence interval $^{\mathrm{a}}$

\begin{tabular}{lll}
\hline & Two years & Five years \\
\hline Overall $^{\mathrm{a}}$ & $0.18(0.10-0.29)$ & $0.49(0.32-0.64)$ \\
No nodal disease & $0.19(0.04-0.42)$ & $0.49(0.09-0.81)$ \\
Nodal disease & $0.18(0.09-0.30)$ & $0.49(0.30-0.65)$ \\
No tumor deposits & $0.00(0.00-0.00)$ & $0.23(0.07-0.46)$ \\
Tumor deposits & $0.31(0.17-0.46)$ & $0.66(0.40-0.83)$ \\
T-category: $\mathrm{pT} 1 / \mathrm{pT} 2$ & $0.07(0.00-0.27)$ & $0.37(0.05-0.72)$ \\
T-category: $\mathrm{pT} 3 / \mathrm{pT} 4$ & $0.22(0.11-0.34)$ & $0.52(0.33-0.68)$
\end{tabular}

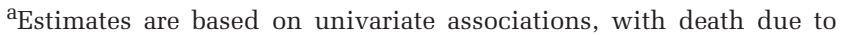
other causes treated as a competing risk.

liver metastases. All four of these latter cases had undergone partial hepatectomy for metastatic debulking, but demonstrated residual liver disease on post-surgical imaging; in three of four cases, they 
were described as 'numerous' or 'multiple' tumors rather than quantified (Figure 2). In contrast, all four patients with nodal and liver disease but not deposits underwent partial or complete hepatectomy for disease; three of them had no residual disease on post-surgical imaging, whereas the fourth demonstrated four remaining stable lesions.

\section{Mesenteric Tumor Deposits and Survival}

Follow-up data was available on all 72 patients (Table 1). Nine (12\%) patients died of disease, with a median survival time of 52 months (range: 2-96). Eleven $(15 \%)$ patients died of causes unrelated to their small intestine neuroendocrine tumors. Twenty-three (32\%) patients were alive with disease, of whom 13 progressed. Among those surviving patients who did not progress, the median (range) follow-up time was 22.2 months (2.3-121.5 months). Progression-free survival subdistribution curves were used to estimate probability of progression-free survival over time for different competing risks by group. These estimates treat progression or death due to disease as the event of interest, with death due to other causes as a competing risk. Those with and without nodal metastasis had similar cumulative incidence of progression and death due to disease; however, those with nodal metastasis at surgery appeared to have lower incidence of death due to other causes ( $P=0.145$, data not shown). The patients with tumor deposits were more likely to progress or die from disease than those without deposits $(P=0.001$, Figure 3$)$.

The cumulative incidence of progression/death due to disease at 2 and 5 years among all patients and among different patient groups was estimated (Table 3). The overall estimated cumulative incidence of progression and death due to disease at 2 years was 0.18 (95\% confidence interval: 0.1, 0.29).

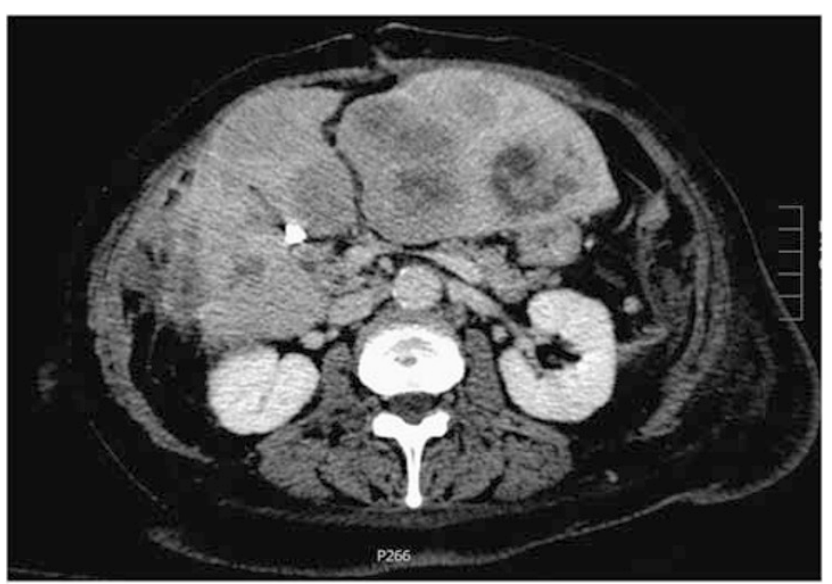

Figure 2 Numerous liver metastases in a small intestine neuroendocrine tumor patient with mesenteric tumor deposits but not lymph node disease (Eovist-enhanced Magnetic Resonance Imaging).
Among patients with deposits at surgery, the 2-year cumulative incidence was $0.31(95 \%$ confidence interval: 0.17, 0.46), whereas no patient without deposits at surgery progressed or died of disease before 2 years. In addition, the 5-year cumulative incidence was 0.66 (95\% confidence interval: 0.40 , 0.83) for those with deposits and $0.23(95 \%$ confidence interval: 0.07 to 0.46 ) for those without deposits. There was strong evidence suggesting that the cumulative incidence subdistributions for progression or death due to disease are different between those with and without tumor deposits $(P=0.001)$.

Table 4 shows the estimated hazard ratios from the progression-free survival Cox model, along with $95 \%$ confidence intervals. There was insufficient evidence to indicate a difference in the rate of progression-free survival between patients with pT3/pT4 disease and those with pT1/pT2 disease (hazard ratio: $1.1 ; 95 \%$ confidence interval: $0.3,3.7$; $P=0.944$ ). However, patients with tumor deposits at

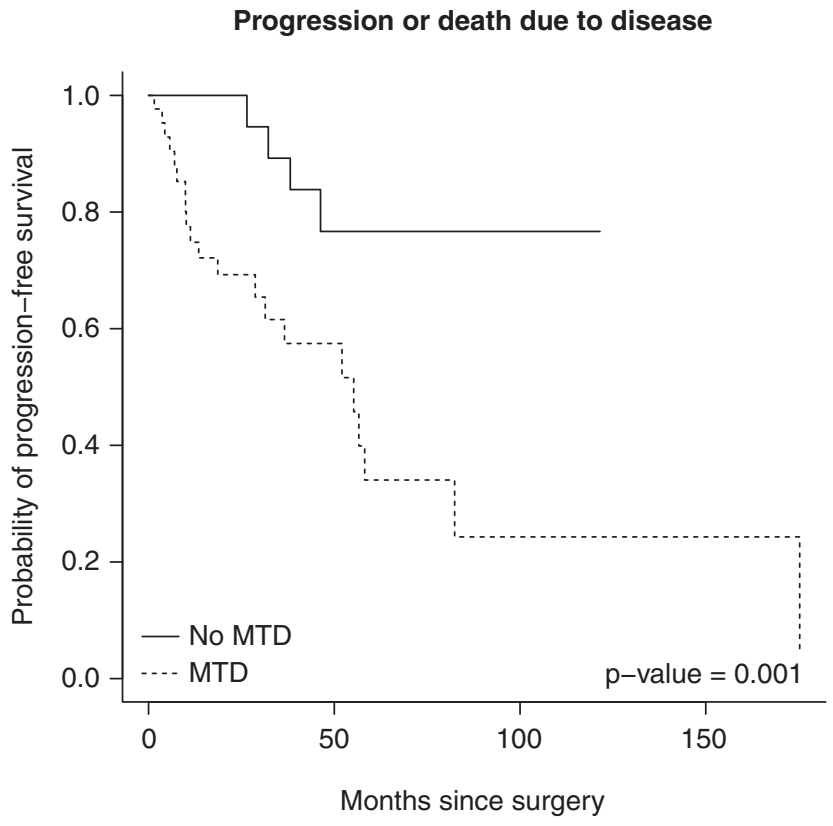

Figure 3 Estimated probability of survival subdistributions (1 cumulative incidence) for progression or death due to disease in patients with and without mesenteric tumor deposits.

Table 4 Parameter estimates from Cox proportional hazards model for progression-free survival, censoring death due to other causes

\begin{tabular}{lclc}
\hline & $\begin{array}{c}\text { Hazard } \\
\text { ratio }\end{array}$ & $\begin{array}{l}\text { Lower/upper } \\
\text { 95\% confidence } \\
\text { interval }\end{array}$ & P-value \\
\hline T-category: pT3/pT4 & 1.05 & $0.29 / 3.74$ & 0.944 \\
Tumor deposits & 4.04 & $1.30 / 12.52$ & 0.016 \\
\hline
\end{tabular}


the time of surgery had 4.0 times the hazard rate (95\% confidence interval: 1.3, 12.5; $P=0.016$ ) associated with progression and death due to disease compared with patients without deposits.

\section{Discussion}

Mesenteric tumor deposits in advanced colorectal carcinoma, which have been variably reported to occur in anywhere from 4.5 to $45 \%$ of patients, ${ }^{16}$ were originally regarded by the American Joint Committee on Cancer staging guidelines as T- or $\mathrm{N}$-category factors, depending first on size and later on contour, ${ }^{16,17}$ although these two criteria are now considered inaccurate and poorly reproducible, respectively. ${ }^{18}$ In the current staging system, tumor deposits fulfill the criterion for pN1c disease, but only if nodal metastases are not otherwise identified. ${ }^{11}$ It is well recognized that they impart a negative prognosis on patients with advanced colorectal carcinoma $^{7-10}$ likely worse than nodal disease $^{7}$ but not as grave as distant metastases. ${ }^{9}$ Various authors at different points in the past 15 years have offered opinions on how best to incorporate tumor deposits into the staging of colorectal carcinoma, usually suggesting that they be a component of $\mathrm{N}$-category ${ }^{17,19}$ or M-category ${ }^{10,19}$ disease. In recent years, Puppa et $a l^{8,18,20}$ have proposed microscopic diagnostic criteria for tumor deposits in colorectal carcinoma and elucidated their role as a negative predictive factor in multivariate analysis. In addition, colorectal carcinoma patients with tumor deposits are more likely to experience local recurrence, ${ }^{16}$ and they develop distant metastases earlier than patients without deposits. $^{10}$

Although Puppa et $a l^{20}$ have reported the identification of tumor deposits in other adenocarcinomas, including gastric and pancreatic, their existence in the context of neuroendocrine tumors, including small intestine neuroendocrine tumors, has been scarcely evaluated in the pathologic literature. Our data showed that tumor deposits were significantly associated with several adverse factors, including lymphovascular invasion, lymph node metastases, liver metastases, and advanced T-category, although apparently not increased Ki67 index. In addition, tumor deposits were significantly associated with an increased cumulative incidence of disease progression and death due to disease, whereas the association between lymph node metastasis and disease progression and death due to the disease was not significant. Furthermore, a percentage of patients with tumor deposits but not nodal disease developed liver metastases (four out of six), supporting the contention that tumor deposits develop in the context of distant metastatic disease. In a comparison between patients with deposits but no nodal disease and patients with nodal disease but no deposits, the former category more often had metastatic disease in the liver after surgical management of liver lesions (4 of $6(68 \%)$ vs 6 of $19(32 \%))$, suggesting that surgical eradication of liver lesions in the context of tumor deposits may be less successful than in the context of nodal disease by itself.

Although tumor deposits in small intestine neuroendocrine tumor may share some similarities with those from colorectal carcinoma, there do exist differences, including being more common in small intestine neuroendocrine tumor patients $(60 \%$ in our study). Most notably, other authors have identified up to three different types of tumor deposits in colorectal carcinoma, based on features such as vascular confinement and surrounding lymphocytes, arguing that the various types have different prognostic implications. ${ }^{20}$ Although we did occasionally observe sparse lymphocytic infiltration around or partial vascular confinement of small intestine neuroendocrine tumor deposits, these findings were never sufficient to allow categorization of lesions, and we feel that at least preliminarily, all deposits in small intestine neuroendocrine tumors represent the same disease process and should not be subdivided. As with colorectal carcinoma deposits, small intestine neuroendocrine tumor deposits must be distinguished from entirely replaced lymph nodes, as well as from embolic tumor within the confines of a lymphovascular space.

The origin of small intestine neuroendocrine tumor deposits is uncertain. Although they are almost always associated with mesenteric nerves, their microscopic appearance suggests that they entrap the nerves, rather than infiltrate and/or emerge from them. Hence, they are not likely a consequence of perineural invasion by the primary tumor, and nerves simply entrapped within tumor deposits should not be misconstrued as perineural invasion by the tumor. As tumor deposits are typically situated next to a large artery, the possibility that they arise secondary to vascular invasion by tumor should be considered. On rare occasion, we did observe deposits appearing to directly extend from tumor cells within a vascular lumen (Figure 1c). Puppa et $a l^{8}$ have suggested that the origin of colonic tumor deposits depends on the type of deposit observed, with deposits with lymphocytes representing a consequence of lymphatic invasion and deposits without lymphocytes resulting from venous invasion. The latter scenario is more likely applicable to small intestine neuroendocrine tumor deposits.

Mesenteric tumor deposits, especially if large, can be detected radiologically during preoperative evaluation. ${ }^{21,22}$ In some instances, mesenteric tumor burden may be the only finding on imaging. ${ }^{23}$ However, the possibility exists that tumor deposits may be mistaken radiologically or macroscopically for diseased lymph nodes and vice versa; indeed, tumor deposits in colorectal carcinoma have been 
described as 'palpable and grossly similar to small lymph nodes' and are often interpreted as such during gross examination, ${ }^{7}$ and surgical studies of small intestine neuroendocrine tumors often seem to equate the term 'mesenteric disease' with the involvement of mesenteric nodes. This is emphasized by the radiologic interpretation of a 'mesenteric mass' in one of the patients in our study that was determined microscopically to be a wholly replaced lymph node. Therefore, final determination of tumor deposits in small intestine neuroendocrine tumors is probably best left to macroscopic and subsequent microscopic examination of surgically resected tissue, to rule out the possibility of lymph node disease or direct extension by tumor. This emphasizes the importance of evaluation of mesenteric surfaces at the time of specimen grossing, as well as awareness of deposits during microscopic examination and the factors that differentiate them from nodal metastases (irregular contour, entrapment of nerves, and lack of prominent surrounding lymphoid tissue). Otherwise, tumor deposits may be interpreted as involved lymph nodes or not included in the diagnostic report, as sometimes occurred in the original pathology reports of the cases we reviewed.

Our study has several limitations. First, this is a retrospective study, and some clinical information may be incomplete. In addition, there was a relatively small number of patients included in the study. Furthermore, a small fraction of patients had no long-term follow-up; of the patients who were disease free and living at last follow-up, $9(25 \%)$ had follow-up of $\leq 1$ year, typically due to recent diagnosis. Lack of longer follow-up reduces the information contained in the data, as unlike for many other malignancies, most patients with small intestine neuroendocrine tumors may survive for many years, and many of them may die from other causes rather than from their neuroendocrine tumor. Still, 5 patients (14\% of surviving patients) had $>5$ years of follow-up. Notwithstanding the short follow-up times in the data, mesenteric tumor deposits did appear to have an impact on patient prognosis. In addition to significant associations with other established indicators of poor prognosis, small intestine neuroendocrine tumor patients with tumor deposits experienced a progression/diseasespecific death rate 4.0 (95\% confidence interval: 1.3, $12,5)$ times higher than patients without deposits, consistent with the hazard ratios of $2.5,4.7$, and 8.0 reported by Ueno et al ${ }^{24}$ for survival for 3 different types of colorectal deposits.

The place for mesenteric tumor deposits in the staging of colorectal carcinoma has changed numerous times in recent years and still may be refined further. Accordingly, our study represents what is hopefully the first step in determining the appropriate classification of tumor deposits in patients with small intestine neuroendocrine tumors. Given the propensity of small intestine neuroendocrine tumor deposits to occur alongside lymph node disease and the evidence that they are a metastatic phenomenon, our preliminary data support its place within the American Joint Committee on Cancer N-classification, perhaps with one tumor deposit filling the same role as one positive lymph node. Prospective studies and experiences from other institutions could allow a more robust analysis in the future.

\section{Acknowledgments}

This project was supported by NIH/NCI P50CA095103 (JRA, GDA, MKW, CS).

\section{Disclosure/conflict of interest}

The authors declare no conflict of interest.

\section{References}

1 Haselkorn T, Whittemore AS, Lilienfeld DE. Incidence of small bowel cancer in the United States and worldwide: geographic, temporal, and racial differences. Cancer Causes Control 2005;16:781-787.

2 Maggard MA, O’Connell JB, Ko CY. Updated population-based review of carcinoid tumors. Ann Surg 2004;240:117-122.

3 Rorstad O. Prognostic indicators for carcinoid neuroendocrine tumors of the gastrointestinal tract. J Surg Oncol 2005;89:151-160.

4 Modlin IM, Lye KD, Kidd MA. 5-Decade analysis of 13,715 carcinoid tumors. Cancer 2003;97:934-959.

5 Modlin IM, Champaneria MC, Chan AK, et al. A threedecade analysis of 3,911 small intestinal neuroendocrine tumors: the rapid pace of no progress. Am J Gastroenterol 2007;102:1464-1473.

6 Strobbe L, D’Hondt E, Ramboer C, et al. Ileal carcinoid tumors and intestinal ischemia. Hepatogastroenterology 1994;41:499-502.

7 Goldstein NS, Turner JR. Pericolonic tumor deposits in patients with $\mathrm{T} 3 \mathrm{~N}+\mathrm{MO}$ colon adenocarcinomas: markers of reduced disease free survival and intraabdominal metastases and their implications for TNM classification. Cancer 2000;88:2228-2238.

8 Puppa G, Maisonneuve P, Sonzogni A, et al. Pathological assessment of pericolonic tumor deposits in advanced colonic carcinoma: relevance to prognosis and tumor staging. Mod Pathol 2007;20:843-855.

9 Lo DS, Pollett A, Siu LL, et al. Prognostic significance of mesenteric tumor nodules in patients with stage III colorectal cancer. Cancer 2008;112:50-54.

10 Al Sahaf O, Myers E, Jawad M, et al. The prognostic significance of extramural deposits and extracapsular lymph node invasion in colon cancer. Dis Colon Rectum 2011;54:982-988.

11 Edge SB, Byrd DR, Carducci MA, Compton CCeds. AJCC Cancer Staging Manual, 7th edn. Springer: New York, NY, 2009.

12 Gray RJ. A class of K-sample tests for comparing the cumulative incidence of a competing risk. Ann Stat 1988;16:1141-1154. 
13 Aalen O. Nonparametric estimation of partial transition probabilities in multiple decrement models. Ann Stat 1978;6:534-545.

$14 \mathrm{R}$ Core TeamR: A language and environment for statistical computing. R Foundation for Statistical Computing, Vienna, Austria. Available from http:// www.R-project.org. (accessed 8 August 2013).

15 Rindi G, Arnold R, Bosman FT, et al. Nomenclature and classification of neuroendocrine neoplasms of the digestive system, In: Bosman FT, Carneiro F, Hruban RH, Theise ND (eds). WHO Classification of Tumours of the Digestive System, 4th edn. IARC Press: Lyon, France, 2010, pp 13-14.

16 Nagtegaal ID, Quirke P. Colorectal tumour deposits in the mesorectum and pericolon; a critical review. Histopathology 2007;51:141-149.

17 Ueno H, Mochizuki H, Shirouzu K, et al. Multicenter study for optimal categorization of extramural tumor deposits for colorectal cancer staging. Ann Surg 2012;255:739-746.

18 Puppa G. Enhanced pathologic analysis for pericolonic tumor deposits: is it worth it? Am J Clin Pathol 2010;134:1019-1021.
19 Tateishi S, Arima S, Futami K, et al. A clinicopathological investigation of 'tumor nodules' in colorectal cancer. Surg Today 2005;35:377-384.

20 Puppa G, Ueno H, Kayahara M, et al. Tumor deposits are encountered in advanced colorectal cancer and other adenocarcinomas: an expanded classification with implications for colorectal cancer staging system including a unifying concept of in-transit metastases. Mod Pathol 2009;22:410-415.

21 Horton KM, Kamel I, Hofmann L, et al. Carcinoid tumors of the small bowel: a multitechnique imaging approach. Am J Roentgenol 2004;182:559-567.

22 Scarsbrook AF, Ganeshan A, Statham J, et al. Anatomic and functional imaging of metastatic carcinoid tumors. Radiographics 2007;27:55-77.

23 Sheth S, Horton KM, Garland MR, et al. Mesenteric neoplasms: CT appearances of primary and secondary tumors and differential diagnosis. Radiographics 2003;23:457-473.

24 Ueno H, Mochizuki H, Hashiguchi Y, et al. Extramural cancer deposits without nodal structure in colorectal cancer: optimal categorization for prognostic staging. Am J Clin Pathol 2007;127:287-294. 